\title{
El caso de los hospitales regionales de alta especialidad en las redes de sevicios en México. Aportes para el análisis
}

\author{
Eugenia del Carmen Sánchez-Romero y Manuel de la Llata-Romero* \\ Dirección General de Coordinación de Hospitales Regionales de Alta Especialidad, Comisión Coordinadora de Institutos Nacionales de Salud y Hospitales de Alta \\ Especialidad, Álvaro Obregón, Ciudad de México
}

\section{«[...] se hace camino al andar». Antonio Machado}

Los hospitales regionales de alta especialidad (HRAE) y el modelo de organización han sido objeto de evaluaciones, auditorías y estudios desde distintos enfoques, en los que se ha puesto en entredicho desde la validez del modelo hasta su integración y desempeño en las redes de servicios. Por ello, es pertinente volver la vista atrás y reflexionar sobre lo ya realizado, los logros, los problemas y su contexto en el periodo comprendido entre los años 2012 y 2018.

Los HRAE son seis organismos públicos descentralizados que cuentan con siete hospitales con entre 5 y 12 años de funcionamiento, por lo que se puede decir que son establecimientos relativamente jóvenes en proceso de consolidación. Además, tienen características particulares para su operación de acuerdo con el contexto estatal y regional en el que se encuentran ubicados.
El proyecto de contar con los HRAE en el Sistema Nacional de Salud se inició con la reforma de la Ley General de Salud ${ }^{1}$, para concretarse después en el Plan Maestro de Infraestructura $2003^{2}$, en el que se definieron 18 regiones en el país y un hospital de alta especialidad, respectivamente.

El modelo conceptual completo de la organización de Ios HRAE fue propuesto de forma detallada en el documento «Innovaciones en gestión hospitalaria en México. El caso de los hospitales regionales de alta especialidad $\gg 3$.

Las modalidades para su operación son dos: la primera, bajo un esquema tradicional de construcción con fondos federales en Chiapas, Oaxaca y la Península de Yucatán; y la segunda, en el modelo de contrato de prestación de servicios', actualmente en asociación público-privada, para la construcción y los servicios de soporte en El Bajío, Ciudad Victoria e Ixtapaluca.

I Los HRAE nacieron de los contratos suscritos bajo el modelo de inversión público-privada; el Hospital Regional de Alta Especialidad del Bajío fue el primero de salud en todo el país. Los contratos para los hospitales regionales de alta especialidad del Bajío, Ciudad Victoria e Ixtapaluca estaban soportados jurídicamente en el acuerdo que establece las reglas para la realización de contratos de prestación de servicios, publicado en el Diario Oficial de la Federación el 09 de abril de 2004 . Disponible en: https://www.gob.mx/cms/uploads/attachment/file/21241/Acuerdo_Reglas_PPS.pdf. Actualmente se denominan asociaciones público-privadas, según la ley publicada el 16 de enero de 2012, la cual está disponible en: http://www.diputados.gob.mx/LeyesBiblio/pdf/LAPP_150618.pdf

\section{Correspondencia:}

*Manuel de la Llata-Romero

Comisión Coordinadora de Institutos Nacionales de Salud y Hospitales

de Alta Especialidad

Periférico Sur, 4118, 1. ${ }^{\text {er }}$ piso

Col. Jardines del Pedregal

C.P. 01900, Álvaro Obregón, Ciudad de México

E-mails: manueldelallata@gmail.com y manuel.dellata@salud.gob.mx

Date of reception: $20-12-2018$

Date of acceptance: 21-12-2018

DOI: 10.24875/HMCM.18000180 
Con estos hospitales se acercó la alta especialidad a la población del interior del país al contar con alternativas para su atención, lo que incrementó la adherencia de los tratamientos y la mejora en los resultados, sin que el paciente y su familia tuvieran que trasladarse a Ciudad de México. Asimismo, se abrieron los campos clínicos para la formación de especialistas y la actualización y capacitación de personal de salud, además del desarrollo de proyectos de investigación.

Se debe considerar que, en algunas de las áreas clínicas, el personal especializado, los insumos y medicamentos con los que cuentan los HRAE son los únicos disponibles en algunas regiones, en contraste con otros hospitales públicos estatales. En consecuencia, la alta especialidad de las grandes urbes puede ser visualizada de manera diferente en el contexto de los diferentes estados.

Por ello es importante mencionar que, sin considerar otro tipo de variable más que su ubicación geográfica, los HRAE brindan la atención de alta especialidad necesaria a la población vulnerable o en condiciones socioeconómicas precarias, por estar ubicados en algunos de los estados con menos índices de desarrollo humano, como Chiapas y Oaxaca, algunas zonas de la Península de Yucatán y los estados de Hidalgo y Veracruz.

Una muestra innegable es que, a pesar de las restricciones, se han ido consolidando las acciones e incrementando el número de población atendida. En el periodo comprendido entre 2013 y 2017 fueron atendidas un total de 887,100 personas, de las cuales un $96 \%$ con niveles socioeconómicos vulnerables, incluyendo atención de población indígena, pacientes migrantes y extranjeros.

La referencia de pacientes por instituciones públicas en 2017 alcanzó porcentajes que van del 98.9\% en el Hospital Regional de Alta Especialidad del Bajío (HRAEB) al 15\% en el Hospital Regional de Alta Especialidad de la Península de Yucatán (HRAEPY), con lo que se pueden observar las marcadas diferencias de integración a las redes de servicios en las distintas regiones. Para agilizar la atención, los HRAE han incorporado a los sistemas de referencia las tecnologías de la información e incluso el uso de aplicaciones en celulares, dependiendo del contexto estatal.

El total de la infraestructura disponible es de 954 camas censables, 530 camas no censables con quirófanos, terapias intensivas, admisión continua y servicios de laboratorio y gabinete. Las camas censables han sido habilitadas en un $72.1 \%$ (688), mientras que las no censables en un $69.2 \%$ (367), lo cual representa un área de oportunidad para brindar atención a la población. En general, las demás áreas, gabinetes y servicios se encuentran funcionando en los siete hospitales.

La imposibilidad de habilitar todas las camas tiene por lo menos dos factores a considerar. El primero es el reducido número de especialistas y subespecialistas formados del que se dispone, además de la poca disponibilidad de éstos a trasladarse a determinados estados, ya que prefieren los grandes centros urbanos para su práctica profesional.

Este factor se ha ido solucionando con la incorporación paulatina de profesionales médicos, de enfermería y paramédicos de reciente egreso o formados en programas académicos en los propios HRAE. Éste ha sido el caso de los especialistas médicos de los institutos nacionales de salud (INS) y hospitales federales de referencia (HFR), que representan cerca del $25 \%$ de los 1,217 médicos adscritos en los HRAE.

El segundo factor a considerar es la falta de plazas, ya que únicamente se cuenta con 6,752 plazas, con una ocupación de la plantilla en agosto de 2018 del 98.5\% $(6,649)$. Sin embargo, este número de plazas no ha sido suficiente por la cantidad de camas totales y, en particular, por las necesidades de las camas no censables, dentro de las que se encuentran las áreas de terapia intensiva.

A pesar de ello, estas limitaciones no han interferido en la aplicación de la política de cero rechazo, ya que se reciben pacientes y usuarios sin tomar en cuenta su condición social o laboral, sobre todo en Chiapas, Oaxaca, Yucatán e Ixtapaluca, estados en los que se cuenta con toda una estrategia de señalización, publicación de información y traductores entre el personal para atender a los pacientes y sus familiares en lenguas indígenas.

Los servicios de atención médica ambulatoria y hospitalaria muestran resultados importantes. De promedio se tiene un porcentaje de ocupación de camas censables habilitadas del $68.4 \%$, con el punto más alto en un $89.0 \%$ y el más bajo en un $52.6 \%$ en septiembre de 2018 . De ellas se producen más de 27,000 egresos anuales, de 
los cuales un promedio del $88.4 \%$ son por mejoría, y se proporcionan cerca de 430,000 consultas externas sobre padecimientos complejos. Un 93.4\% de los usuarios de los servicios hospitalarios muestran un $80 \%$ de satisfacción en la atención recibida y un $92.0 \%$ en lo referente a la atención ambulatoria.

Se realizan el 81.1 , el 54.5 y el $77.5 \%$ de sesiones de rehabilitación, procedimientos diagnósticos y terapéuticos ambulatorios de alta especialidad, respectivamente, y se priorizan los procedimientos más costo-efectivos con los que no se cuenta en los hospitales estatales.

Como parte de la gestión en calidad de los servicios, los HRAE están acreditados para ofrecer atención en las enfermedades establecidas para el Fondo de Protección contra Gastos Catastróficos, el Seguro Médico Siglo XXI y algunas específicas del Catálogo Universal de Servicios de Salud de acuerdo a la cartera de servicios. Actualmente, se tienen más de 109 servicios acreditados o reacreditados, lo cual ha permitido atender a los pacientes y que ello no les afecte, y tampoco a sus familias, al estar cubiertos los procedimientos, m-amentos y otros insumos por parte de estos fondos, creando círculos virtuosos.

Además, todos los HRAE cuentan con programas para la certificación de establecimientos de atención médica, con diversos grados de avance. El HRAEPY fue el primer HRAE certificado por el Consejo de Salubridad General de 2014, y en 2018 estaba en espera de fecha para la evaluación para recertificación. El HRAEB obtuvo en agosto de 2018 la certificación por tres años y el Hospital Regional de Alta Especialidad de Ciudad Victoria está en espera de fecha para la evaluación.

Un avance importante es el manejo de los m-amentos e insumos, con modelos considerados dentro de las mejores prácticas, siendo el más adelantado el Centro Integral de Servicios Farmacéuticos del Hospital Regional de Alta Especialidad de Ixtapaluca (HRAEI). Todos Ios HRAE han implementado el reporte y seguimiento de los eventos adversos, y en la mayoría se lleva a cabo un seguimiento específico de la idoneidad de las prescripciones.

Como parte del proyecto institucional y del proceso de maduración, los HRAE se han posicionado también como centros de formación de especialistas. Desde 2012-2013 seis HRAE cuentan con cursos de formación médica, y en el ciclo académico de 2015 el HRAEl inició dichos programas, con lo que en la actualidad los siete hospitales forman recursos humanos, con cerca del $95 \%$ de eficiencia terminal. Se imparten programas tanto de entrada directa como indirecta, algunos de ellos dentro del Programa Nacional de Posgrados de Calidad del Consejo Nacional de Ciencia y Tecnología (CONACyT).

Se tienen inscritos 370 residentes nacionales y extranjeros en cursos de residencias y de alta especialidad. Se han tenido más de 200 egresados, de los cuales un $50 \%$ se han certificado por consejos e incorporado a instituciones de las regiones, lo cual favorece la distribución de especialistas en los estados, o han continuado su formación en las más prestigiadas instituciones del país.

Los HRAE brindan actualización para el personal de salud (en promedio más de 60 cursos anuales y otros eventos académicos para las redes de servicios), con temas de importancia para la salud de las regiones en las que se encuentran ubicados.

Realizan investigación, tanto por el personal con plaza de investigador (26 investigadores en los 7 hospitales) como por el personal médico y paramédico, y estas acciones se han fomentado y vinculado con las prioridades de salud, tal como ocurre en los INS y HFR. Los investigadores se evalúan tanto dentro del sistema de la propia Secretaría de Salud como en el Sistema Nacional de Investigadores, por lo que el $65.3 \%$ de ellos está dentro del grupo de investigadores de alto nivel.

En 2017 se produjeron un 47.6\% de artículos publicados en revistas consideradas de alto impacto a nivel internacional, con 1.1 artículos de este tipo y 1.8 productos (tesis, artículos, capítulos de libros, presentaciones, entre otros) por investigador; y el $80.6 \%$ son en colaboración con prestigiosas instituciones de investigación científica nacionales e internacionales. También se cuenta con proyectos propuestos para realizar la traslación del conocimiento.

Además de todo ello, la producción científica se encuentra enmarcada en las líneas de investigación que, en general, se vinculan con las prioridades, entre las que destacan: las enfermedades emergentes infecciosas -como dengue, virus del Zika, chikungunya e influenza-, conservación de tejidos para trasplantes, 
genómica de obesidad, tratamientos contra el cáncer, genómica del virus del papiloma humano, problemas renales, oftálmicos y de otro tipo relacionados con las condiciones geográficas, micosis, prevención de riesgos en el embarazo, trasplantes y sobrevida.

A modo de conclusión podemos afirmar que el modelo no es perfecto, pero las contribuciones a la atención en las regiones que albergan los HRAE son importantes. El futuro alberga enormes dificultades y los retos a enfrentar son muy grandes; sin embargo, se cuenta con una masa crítica capaz y comprometida con el proyecto de los HRAE, tanto en las áreas directivas como en las operativas, que han integrado en la atención y aumentado la capacidad resolutiva de las redes locales, y todo ello apoyado en la formación de especialistas que se arraigan y permanecen en las regiones, actualización y capacitación del personal de salud con programas orientados a las necesidades y, de manera muy importante, en el desarrollo de investigación en prioridades regionales de salud para beneficio de la población.

\section{AGRADECIMIENTOS}

Agradecimientos a la Mtra. Ana Cecilia Flores Naranjo y a la C.P. María Guadalupe Rico Puebla por su valiosa ayuda en la integración de este documento.

\section{BIBLIOGRAFÍA}

1. Poder Ejecutivo Federal. Ley General de Salud, Artículo 77 bis 5 fracción II, que define la responsabilidad del Estado mexicano de proporcionar servicios en unidades de alta especialidad. Disponible en: http://www.salud. gob.mx/cnts/pdfs/LEY_GENERAL_DE_SALUD.pdf

2. Disponible en: http///saludchiapas.gob. $\mathrm{mx} / \mathrm{doc} / \mathrm{marco}-$ normativo/federal/ planes-y-programas/6.- $\% 20$ Plan $\% 20$ Maestro $\% 20$ Infraestructura $\% 20$ en $\% 20$ Salud.pdf

3. SSA. Documento de IOS HRAE DGPlaDeS-SSa. Innovaciones en gestión hospitalaria en México. El caso de los hospitales regionales de alta especialidad (HRAE). México, D.F.; 2006. 